\title{
Acknowledgment to Reviewers for 2014
}

On behalf of the editor and editorial board, we would like to express our sincere gratitude for the reviewers and contributors who have generously endowed with their time and efforts in appraising the manuscripts submitted to Clinical and Experimental Reproductive Medicine in 2014.

It was their help that made it possible for the journal to achieve a new level of caliber and rigor.

\begin{tabular}{|c|c|c|c|}
\hline Chae, Hee Dong & Ulsan University & Kim, Sunmie & Seoul National University \\
\hline Cheon, Yong-Pil & Sungshin Women's University & Kim, Yong-Jin & Korea University \\
\hline Chi, Heejun & Mizmedi Hospital & Kim, You-Shin & CHA University \\
\hline Cho, Moon-Kyoung & Chonnam National University & Ko, Chemyong & University of Illinois at Urbana-Champaign, \\
\hline Cho, Yeon-Jean & Dong-A university & & College of Veterinary Medicine \\
\hline Choi, Doo-Seok & Sungkyunkwan University & Kwak, Joanne YH & Rosalind Franklin University of Medicine and Science \\
\hline Choi, Inhong & YonseiUniversity & Lee, Dong Yoon & Sungkyunkwan University \\
\hline Choi, Won-Jun & Gyeongsang National University Hospital & Lee, Hyoung-Song & Cheil General Hospital \\
\hline Choi, Young Sik & Yonsei University & Lee, Joongyeup & Hamchoon Women's Clinic \\
\hline Choi, Youngsok & CHA University & Lee, Kyung Wook & Korea University \\
\hline Chun, Sang-Young & Chonnam National University & Lee, Kyung-Ah & CHA University \\
\hline Chun, Sungwook & Inje University & Lee, Sung Ki & Konyang University \\
\hline Chung, Mikyung & Seoul Rachel Fertility Center & Lim, Hyunjung Jade & KonkukUniversity \\
\hline Gye, Myung Chan & Hanyang University & Oh, Jeong Su & Sungkyunkwan University \\
\hline Han, Myoungseok & Dong-A University & Park, Chan Woo & Catholic Kwandong University \\
\hline Hur, Sungeun & Konyang University & Park, Dong-Wook & Kwandong University \\
\hline Hwang, Kyuri & Seoul National University & Park, Hyun-Tae & Korea University \\
\hline Jee, Byung Chul & Seoul National University & Park, Joon Cheol & Keimyung University \\
\hline Joo, Bo-Sun & Good Moonhwa Hospital & Ryu, Hyun Mee & Kwandong University \\
\hline Joo, Jong Kil & Pusan National University & Seo, Ju Tae & Catholic Kwandong University \\
\hline Jun, Jin Hyun & Eulji University & Seo, Seok-Kyo & Yonsei University \\
\hline Jung, Kyung Ah & Ewha Womans University & Song, Haengseok & CHA University \\
\hline Kim, Chung-Hoon & Ulsan University & Song, In Ok & Catholic Kwandong University \\
\hline Kim, Gi Jin & CHA University & Song, Seung Hun & CHA University \\
\hline Kim, Hoon & Seoul National University & Yang, Kwang Moon & Catholic Kwandong University \\
\hline Kim, Hye Ok & Catholic Kwandong University & Yoon, Ho & CHA University \\
\hline Kim, Jong Hyun & MizMedi Hospital & Yoon, Sook-Young & CHA University \\
\hline Kim, Soo Woong & Seoul National University & Youm, Hyewon & Seoul National University Bundang Hospital \\
\hline
\end{tabular}

This is an Open Access article distributed under the terms of the Creative Commons Attribution Non-Commercial License (http://creativecommons.org/licenses/by-nc/3.0/) which permits unrestricted non-commercial use, distribution, and reproduction in any medium, provided the original work is properly cited. 